\title{
DISTINCT EXPRESSION PROFILE AND HISTOLOGICAL DISTRIBUTION OF NLRP3 INFLAMMASOME COMPONENTS IN THE TISSUES OF HAINAN BLACK GOAT SUGGEST A SITE-SPECIFIC ROLE IN THE INFLAMMATORY RESPONSE
}

\author{
Kaizhao ZHANG, Pan TAO, Jianxin LiU, Qingnan WANG, Shikun GE \\ and Zhangyong NING ${ }^{*}$ \\ College of Veterinary Medicine, South China Agricultural University, \\ Guangzhou 510642, People's Republic of China
}

(Received 21 December 2016; accepted 4 April 2017)

\begin{abstract}
The NOD-like receptor protein 3 (NLRP3) inflammasome comprised of NLRP3, ASC and caspase-1 plays an important role in the inflammatory and innate immune response. However, little is known about the expression pattern and histological distribution of these genes in goat. Here, we first cloned the fulllength cDNAs of the NLRP3, ASC and caspase-1 genes of Hainan black goat and produced their polyclonal antibodies. Tissue-specific expression and histological distribution of these genes were analysed. Phylogenetic analysis revealed that these three goat genes had high homology with Bos taurus genes and low homology with avian or fish genes. After immunisations with these recombinant Histagged proteins, the titres of antiserum were higher than 1:1024 and purified IgG was obtained. These three genes were expressed in all examined tissues, the mRNA expression level of NLRP3 and caspase-1 was most abundant in the spleen and mesenteric lymph nodes (MLNs), while ASC was primary expressed in the liver, spleen and kidney. The histological distribution of NLRP3, ASC and caspase-1 was detected in myocardial cells, hepatocytes, focal lymphocytes, bronchiolar epithelial cells, renal tubular epithelial cells, cortical neurons and endothelial cells of the germinal centres in the MLNs. These results will be helpful in further investigations into the function of the NLRP3 inflammasome and in elucidating its role in caprine inflammatory diseases.
\end{abstract}

Key words: Goat, NLRP3, ASC, caspase-1, expression, tissue distribution

As part of the immune response to infection, inflammation is initiated on recognising the signs of tissue injury or disturbances of the host steady state which could result in tissue damage (Davis et al., 2011; Horvath et al., 2011; Latz et al., 2013). The innate immune system, which establishes the first line of host defence, plays an important role in the process of inflammation and regulates the generation of long-lived adaptive immune responses (Ferrero-Miliani et

*Corresponding author; E-mail: ningzhyong@scau.edu.cn; Phone/Fax: 0086 (20) 8528-0234 
al., 2007). Activation of the innate immune system relies on pattern recognition receptors (PRRs), such as NOD-like receptors (NLRs), RIG-I like receptors, Toll-like receptors and C-type lectin families. NLRs and their recruited downstream proteins can assemble into large multiprotein complexes (inflammasomes), leading to the production of important soluble pro-inflammatory substances which participate in the development of inflammation (Kumar et al., 2009; Takeuchi and Akira, 2010; Davis et al., 2011; Mahla et al., 2013). NLRP3 inflammasome, which is composed of NOD-like receptors 3 (NLRP3), adaptor apoptosisassociated speck-like protein (ASC) and caspase-1 (Sutterwala et al., 2014), plays important roles in inflammatory and innate immune responses associated with autoimmune/inflammatory diseases (Federici et al., 2012; Chang, 2013; VeraLastra et al., 2013), as well as with a variety of dissimilar agonists such as influenza virus, Candida albicans, prions, ATP, bacterial RNA and uric acid (Mariathasan et al., 2006; Kanneganti et al., 2006; Martinon et al., 2006; Allen et al., 2009; Joly et al., 2009; Shi et al., 2012). The NLRP3 inflammasome has a pivotal function in regulating the cleavage and maturation of pro-IL-1 $\beta$ and pro-IL18 , and ultimately results in an inflammatory response and a programmed cell death, pyroptosis (Lamkanfi, 2011; Sutterwala et al., 2014). However, an overactive NLRP3 inflammasome can induce aberrant IL-1 $\beta$ secretion which is harmful to the host and associated with various inflammatory lesions.

Comprehensive analysis of expression profiles and histological distribution is crucial for elucidating the functions of the NLRP3 inflammasome in inflammatory responses and innate immunity. To date, research concerning the NLRP3 inflammasome has been concentrated on humans, mice, pigs and chickens (Anderson et al., 2004; Kummer et al., 2007; Tohno et al., 2011; Huang et al., 2014; Ye et al., 2015). Basic data on the sequence, expression pattern and histological distribution of NLRP3, ASC and caspase-1 are still unknown in goat. China has the largest goat population in the world (Wu et al., 2009; Wang et al., 2015). The Hainan black goat, a meat-type goat breed raised in South China, has good heat resistance characteristics (Wang et al., 2014). In recent years, with the increase in farm size and the number of goats bred, inflammatory diseases have exerted increasing influence on the production of Hainan black goat (Konishi et al., 2004; Venkatesan et al., 2010; Li et al., 2012; Duan et al., 2014; Zhang et al., 2016). Infections causing inflammation in goats have become difficult to treat because the basic features of the inflammatory response are unknown.

In this work, we first cloned full-length cDNAs of the NLRP3, ASC and caspase-1 genes of Hainan black goat by polymerase chain reaction (PCR) and produced their polyclonal antibodies by the immunisation of rabbits using purified recombinant prokaryotic expression proteins. Tissue-specific expression and histological distribution of NLRP3, ASC and caspase-1 were analysed by realtime quantitative PCR and immunohistochemical detection. The results should 
be helpful in further investigations into the function of the NLRP3 inflammasome and should elucidate its role in specific tissues involved in caprine inflammatory diseases. Also, they can serve as basic data for the prevention and treatment of inflammatory diseases in goat.

\section{Materials and methods}

Sample collection and tissue preparation

Nine healthy Hainan black goats (6 months old with body weights of $22.5 \pm$ $1.6 \mathrm{~kg}$ ) were obtained from a purebred herd in a local livestock farm in the city of Zeng-cheng, Guangdong province. The animal experiments were approved by the Institutional Animal Care and Use Committee of South China Agricultural University. After anaesthesia and euthanasia, tissue samples including the heart, brain, liver, spleen, lung, kidney and mesenteric lymph nodes (MLNs) were collected promptly. Each tissue was separated into two parts: one was frozen in liquid nitrogen for RNA extraction and the other fixed in $10 \%$ neutralised buffered formalin for immunohistochemical detection.

\section{RNA extraction, first-strand cDNA synthesis and RT-PCR}

Total RNA was isolated from the collected samples using the RNAiso Plus reagent (Takara Biotech, Dalian, China) in accordance with the manufacturer's instructions. The concentrations of total RNA were measured using a NanoDrop 2000 spectrophotometer (Thermo Fisher Scientific, USA). One $\mu \mathrm{g}$ of total RNA was converted to first-strand cDNA using a PrimeScript RT reagent with gDNA eraser (Takara Biotech, Dalian, China) according to the manufacturer's instructions. Three sets of primer pairs were designed and synthesised on the basis of the Capra hircus NLRP3 (XM_005682796.2), ASC (XM_005697733.2) and caspase-1 (XM_013969391.1) mRNA sequences in GenBank. The gene specific primers were as follow: NLRP3 - Fw: 5'-AGGACTCTGACCAGGTCT GCA-3', NLRP3 - Rv: 5'-GTGTTTCCGCCACTACCGA-3', ASC - Fw: 5'-ATG GGGTGCACACGCGAT-3', ASC - Rv: 5'- TCAGCTCTGCTCCAGGTCGTC-3'; caspase-1 - Fw: 5'-CAAGGCTAAGAAGGGGAAGT-3', and caspase-1 - Rv: 5'-TAGGGGTCATGCACTATC-3'. The genes were amplified by polymerase chain reaction (PCR) in a $50-\mu 1$ reaction volume with the following conditions: denaturation at $95^{\circ} \mathrm{C}$ for $5 \mathrm{~min} ; 36$ cycles of denaturation at $95^{\circ} \mathrm{C}$ for $30 \mathrm{sec}$, annealing at $55^{\circ} \mathrm{C}$ for $35 \mathrm{sec}$ for NLRP3 and caspase- 1 , and at $57^{\circ} \mathrm{C}$ for $35 \mathrm{sec}$ for ASC, and extension at $72{ }^{\circ} \mathrm{C}$ for $3 \mathrm{~min} 30 \mathrm{~s}$ for NLRP3, for 2 min for caspase-1 and for $55 \mathrm{sec}$ for ASC, followed by a final extension cycle at $72{ }^{\circ} \mathrm{C}$ for $10 \mathrm{~min}$. The PCR products purified by the Agarose Gel DNA Extraction Purification kit Ver4.0 (Takara Biotech, Dalian, China) were cloned into pMD18-T vector (Takara Biotech, Dalian, China) and sequenced, respectively. 


\section{Real-time quantitative PCR}

The mRNA expressions level of NLRP3, ASC and caspase-1 were measured by real-time quantitative PCR using the SYBR ${ }^{\circledR}$ Premix Ex Taq ${ }^{\mathrm{TM}}$ II $($ Perfect Real Time) (Takara Biotech, Dalian, China) with a CFX96 Real-Time PCR Detection System (Bio-Rad, Hercules, CA). The PCR amplification conditions used were: $95{ }^{\circ} \mathrm{C}$ for $30 \mathrm{sec}$, followed by 40 cycles of $95^{\circ} \mathrm{C}$ for $5 \mathrm{sec}$ and $60^{\circ} \mathrm{C}$ for $30 \mathrm{sec}$. The gene-specific primers used were as follows: NLRP3 - Fw: 5'CCGTCTGGGTGAGAGCGTGAA-3', NLRP3 - Rv: 5'-TCCTGTTGGCTCCTGTGTTCCT-3'; ASC - Fw: 5'-GCCGTGGACCTTACCGACAA-3', ASC - Rv: 5'-GCAGTCCTGGCTTGGCTATCTT-3'; caspase-1 - Fw: 5'-GGATACAATAAATGGCTTGCTGG-3', caspase-1 - Rv: 5'-CTCGGGCTTTATCCATAGTTGT-3'; $\beta$-actin - Fw: 5'-AGGCAGTGTCGGTCATCGT-3', and $\beta$-actin - Rv: 5'-CCTCAGGTCATCATCACGGAAG-3'. The relative expression levels of these genes were calculated by normalising the levels of gene transcripts to that of endogenous housekeeping gene $\beta$-actin transcripts using a relative standard curve method through the $2^{-\Delta \Delta \mathrm{Ct}}$ formula (Livak and Schmittgen, 2001). All samples were performed in triplicate. Statistical analysis was carried out by using independent sample (two-tailed) $t$-test and one-way ANOVA test using SPSS software (version 20.0), and the figure was made by GraphPad Prism (GraphPad Software, La Jolla, CA). Data are presented as mean \pm standard deviation (SD). A P value $<0.05$ was considered to be statistically significant.

Expression, purification and Western blot detection of recombinant NLRP3, ASC and caspase-1

The expression primers of goat NLRP3, ASC and caspase-1 were as follow: NLRP3 - Fw: (Sal I) 5'-CCGACCGTCGACTGATGATCATGGCAA-3', NLRP3 - Rv: (Not I) 5'-ATTATAGCGGCCGCCTACCGAGAAGGCT-3'; ASC - Fw: (EcoR I) 5'-TACCCGGAATTCATGGGGTGCACACGC-3', ASC Rv: (Xho I) 5'-TCTTCGCTCGAGTCAGCTCTGCTCCAG-3'; caspase-1 - Fw: (Sac I) 5'-CTAGAAGAGCTCATGGCCGACAAGG T-3', and caspase-1 Rv: (Xho I) 5'-CGCCGCCTCGAGTTAATATCCTGGAA-3' (underlined nucleotides indicate restriction enzyme sites on each primer). Expression and purification of these recombinant proteins and Western blot were performed as reported by us previously (Zhou et al., 2013). Briefly, NLRP3, ASC and caspase-1 PCR products were digested by corresponding two enzymes, and ligated into the expression vector pET-32a (+) previously digested with the same two enzymes, respectively, to form a sequence encoding a fusion protein of NH2-terminal Histag. These recombinant plasmids were sequenced on both strands and termed as pET-32a (+)-NLRP3, pET-32a (+)-ASC and pET-32a (+)-caspase-1, respectively. The competent E. coli BL21 (DE3) cells (Dingguo, Beijing, China) were transfected with the recombinant plasmid. A single colony was cultured in Luria- 
Bertani (LB) medium containing $100 \mu \mathrm{g} / \mathrm{ml}$ ampicillin and grown at $37^{\circ} \mathrm{C}$ to an optical density of 0.6 , and then $1 \mathrm{mM}$ isopropyl $\beta$-D-1-thiogalactopyranoside (IPTG) was added and the culture was incubated at $37^{\circ} \mathrm{C}$ for $5 \mathrm{~h}$. According to the manufacturer's protocols, the inclusion body protein was successfully purified by a His-tagged inclusion protein purifying kit (Cwbiotech, Beijing, China). These purified His-tagged infusion proteins were analysed by Western blot with an anti-6X His tag chicken antibody (ab3553; Abcam, Cambridge, UK) and the purified IgG prepared by us.

\section{Antiserum preparation and $\operatorname{Ig} G$ purification}

Antiserum preparation against NLRP3, ASC and caspase-1 and IgG purification were performed as described by us previously (Zhou et al., 2013; Ye et al., 2015). Briefly, New Zealand white rabbits were immunised using $50 \mu \mathrm{g}$ of a recombinant protein mix with complete Freund's adjuvant for initial immunisation at the first two times and with incomplete Freund's adjuvant for subsequent boost at the other three times, at 8-day intervals. Then the antiserum was detected by indirect ELISA and IgG was prepared using a Rapid Antibody Purification Kit (Cell Biolabs Inc., Shanghai, China).

\section{Immunohistochemical detection}

Immunohistochemical detection was performed as described in our previous reports (Zhou et al., 2013; Huang et al., 2014; Ye et al., 2015). Briefly, the sections were incubated with rabbit purified anti-NLRP3, anti-ASC or anticaspase-1 purified IgG diluted 400 -fold at $37^{\circ} \mathrm{C}$ for $2 \mathrm{~h}$. After incubation with the appropriate HRP-conjugated mouse anti-rabbit IgG antibody (Cell Signaling Technology, Beverly, MA, USA) diluted 2,000-fold at $37^{\circ} \mathrm{C}$ for $1 \mathrm{~h}$, the slices were washed three times with PBS at 5-min intervals. For visualisation, the DAB kit (ZSGQ-BIO, China) was used and counterstained with haematoxylin according to the manufacturer's guidelines. Finally, the sections were dehydrated with graded ethanol, vitrified by dimethylbenzene, and deposited in neutral balsam. Images were obtained with a Leica DM4000 B LED microscope equipment (Leica Microsystems, Germany).

\section{Results}

Sequence analysis of NLRP3, ASC and caspase-1 genes in goat

The coding nucleotide sequence of NLRP3, ASC and caspase-1 was amplified by RT-PCR and the sequences were deposited in GenBank (KM236558 for NLRP3, KM576768 for ASC and KP999980 for caspase-1). The protein deduced on the basis of these nucleotide sequences is composed of 1,031 amino acids for NLRP3, 196 amino acids for ASC and 403 amino acids for caspase-1. 
The molecular weights of NLRP3, ASC and caspase-1 proteins were predicted to be $117.88023,22.0967$, and $45.33649 \mathrm{KDa}$, respectively. The isoelectric points (PI) predicted using the ProtParam tool (http://web.expasy.org/protparam/) are $6.40,5.34$ and 7.05 , respectively.

The nucleotide sequences of NLRP3, ASC and caspase- 1 were aligned using the MEGA 5.0 software (Fig. 1). The nucleotide homology between goat NLRP3 and that of Bos taurus (NM_001102219), Sus scrofa (NM_001256770), Homo sapiens (NM_004895), Macaca mulatta (EU344966), Callithrix jacchus (GAMR01008031), Mus musculus (NM_145827), Rattus norvegicus (NM_0011 91642) and Chinese Yellow Chicken (KF318520) was 97.5\%, 90.1\%, 82.6\%, $83.9 \%, 83.1 \%, 78.1 \%, 79.4 \%$ and $42.7 \%$, respectively (Fig. 1A). The nucleotide homology between goat ASC and that of Bos taurus (NM_174730), Sus scrofa domesticus (GBZA01000528), Homo sapiens (AF310103), Macaca mulatta (NM_001194572), Mus musculus (NM_023258), Rattus norvegicus (NM_172322), Xenopus laevis (NM_001092919) and Danio rerio (NM_131495) was 94.6\%, $86.1 \%, 79.9 \%, 79.1 \%, 73.2 \%, 72.1 \%, 61.1 \%$ and $36.6 \%$, respectively (Fig. 1B). The nucleotide homology between goat caspase-1 and that of Equus caballus (NM_001081842), Sus scrofa (NM_214162), Bos taurus (XM_592026), Homo sapiens (NM_033292), Callithrix jacchus (XM_009006697), Mus musculus (NM_009807), Rattus norvegicus (NM_012762), Felis catus (NM_001009365), Gallus gallus (AF031351) and Xenopus laevis (NM_001087754) was 84.1\%, $86.3 \%, 97.4 \%, 79.1 \%, 76.6 \%, 72.0 \%, 71.1 \%, 61.2 \%, 50.3 \%$ and $51.3 \%$, respectively (Fig. 1C).

\section{NLRP3, ASC and caspase-1 mRNA expression levels in various tissues}

The mRNA of NLRP3, ASC and caspase-1 was expressed in all examined tissues (Fig. 2). The relative expression level of NLRP3 in the spleen was significantly higher than in the heart, liver, lung, kidney, brain and mesenteric lymph nodes $(\mathrm{P}<0.01)$ (Fig. 2A). ASC expression levels were higher in the liver, spleen, kidney and brain, and lower in the heart, lung and mesenteric lymph nodes (Fig. 2B). Caspase-1 expression levels were highest in the liver and spleen followed by the mesenteric lymph nodes (MLNs) and lung, and lower in the heart, brain and kidney (Fig. 2C).

Expression plasmid construction of NLRP3, ASC and caspase-1 and purification of recombinant proteins

To confirm whether the expression plasmid pET-32a (+)-NLRP3, pET32a (+)-ASC, and pET-32a (+)-caspase- 1 was successfully constructed, PCR and double enzyme digestion analysis were performed. The expression plasmids for NLRP3, ASC and caspase-1 were verified by digestion with SalI and NotI for NLRP3, EcoRI and XhoI for ASC, and SacI and XhoI for caspase-1, respectively, and confirmed by sequencing. 

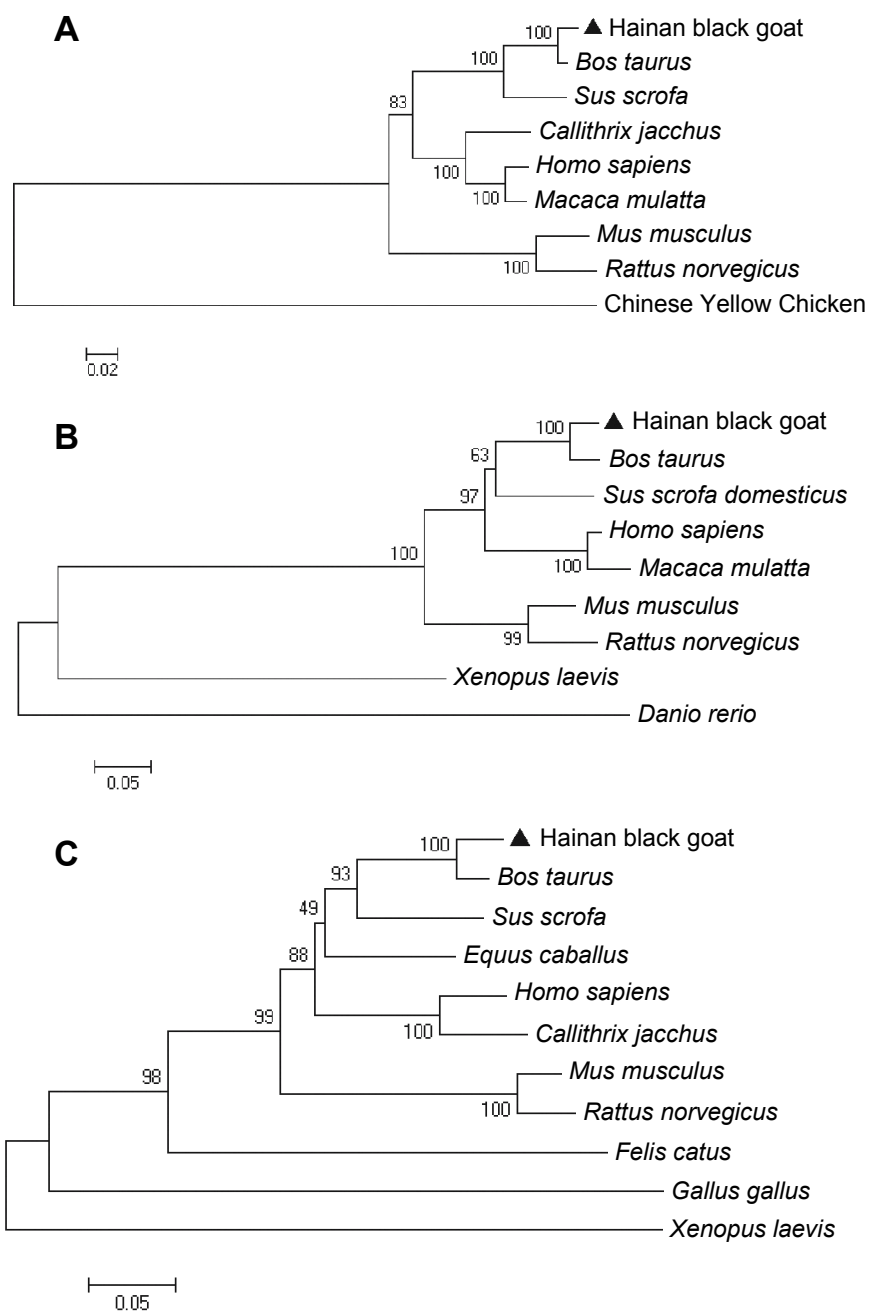

Fig. 1. Phylogenetic analysis of NLRP3 (A), ASC (B), and caspase-1 (C). The phylogenetic tree was conducted in MEGA 5.0 using the neighbour-joining method. Bootstrap values (\%) are represented at each tree node

These expression recombinant plasmids were transformed into E. coli BL21 (DE3). After induction with $1 \mathrm{mM}$ IPTG, the recombinant His-tagged NLRP3, ASC and caspase-1 proteins were effectively expressed (Figs 3A, 4A and 5A). SDS-PAGE showed that the recombinant proteins were the purified proteins consistent with the predicted size (Figs 3B, 4B and 5B). Western blot using His-tag antibody and our prepared $\mathrm{IgG}$ indicated that the fusion proteins were His-tagged NLRP3, ASC and caspase-1 (Figs 3C, 4C, and 5C). 
A

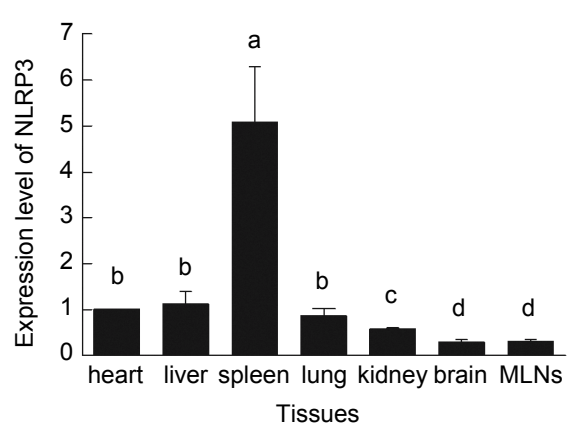

B

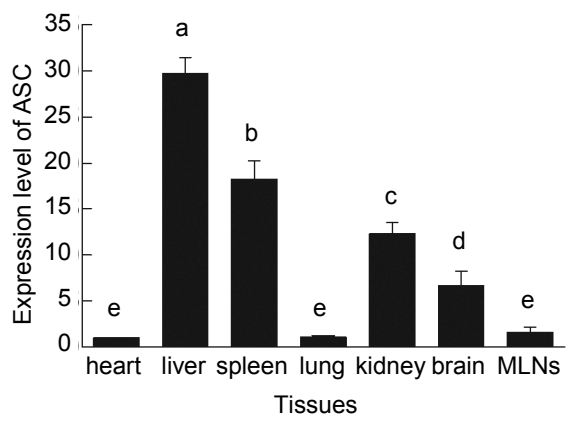

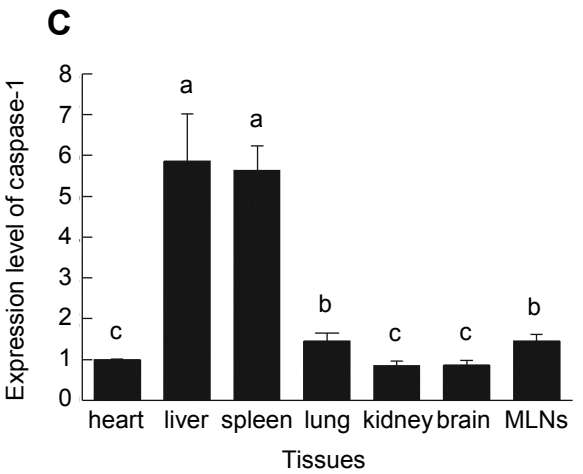

Fig. 2. Relative expression levels of NLRP3 (A), ASC (B), and caspase-1 (C) in various tissues of Hainan black goat. The expression levels were normalised to $\beta$-actin and quantified using the $2^{-\Delta \Delta \mathrm{Ct}}$ formula. All samples were performed in triplicate. The different letters stand for significant difference $(\mathrm{P}<0.01)$, while tissues with the same letter show no difference $(\mathrm{P}>0.05)$.

Data are presented as mean \pm standard deviation (SD)

\section{Antiserum preparation and $\operatorname{Ig} G$ purification}

After immunisation with purified recombinant NLRP3, ASC and caspase-1, antisera with titres higher than 1:1024 as determined by ELISA were collected from New Zealand white rabbits. Purified IgG was obtained from these antisera. Western blot analysis showed that the IgG could specifically recognise the purified proteins (Figs 3D, 4D and 5D).

\section{Immunohistochemical detection of NLRP3, ASC and caspase-1}

Immunohistochemical analysis showed the histological distribution of NLRP3, ASC and caspase-1 proteins in the tissues of Hainan black goats. In the heart, the myocardial cells exhibited moderate to strong positive staining for NLRP3, ASC and caspase-1, while staining of the connective tissues was negative (Fig. 6A-C). In the liver, almost all hepatocytes were strongly positive in the 
cytoplasm and partially positive staining appeared in the nuclei of hepatocytes, while the endothelial cells of the hepatic sinus were moderately positive for NLRP3 and ASC and weakly positive for caspase-1 (Fig. 6D-F). In the spleen, the lymphocytes were weakly positive and the endothelial reticular cells were moderately positive for NLRP3 protein expression (Fig. 6J). However, the endothelial reticular cells and the focal lymphocytes in the splenic corpuscle showed moderate or medium to strong positive staining for ASC and caspase-1 (Fig. 6H and I). In the lung, cells of the bronchiolar epithelial cells stained strongly positive for NLRP3, ASC and caspase-1, while the alveolar epithelial cells of the pulmonary alveoli exhibited weak to medium positive staining (Fig. 6J, K and L). In the kidney, the renal tubular epithelial cells showed strong positivity for NLRP3, ASC and caspase-1 while cells in the glomerulus and the Bowman's capsules stained negative (Fig. 6M, N and O). In the brain, the NLRP3-positive cells included some neurons in the cerebral cortex which showed medium to strong staining, while the matrix exhibited very weak positive staining (Fig. 6P). The ASC-positive cells were primarily cortical neurons with strong positivity in the matrix (Fig. 6Q). For caspase-1 in the brain, the cortical neurons exhibited weak to moderate positivity with negative staining of the matrix (Fig. 6R). In the mesenteric lymph nodes, endothelial cells of the germinal centre showed strong positivity for NLRP3, ASC and caspase-1. Moreover, a strong positive staining for caspase-1 was also found in the diffuse lymphoid tissue (Fig. 6S, T and U).

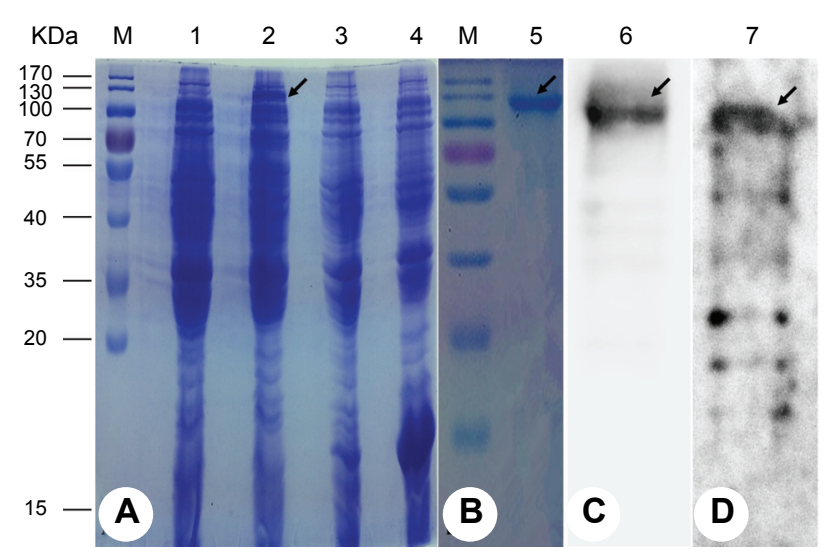

Fig. 3. Analysis of the His-tagged NLRP3 protein expressed in E. coli BL21 (DE3). A: Lane M, the protein marker; Lane 1, total protein from E. coli BL21 (DE3) transformed with plasmid pET-32a (+)-NLRP3 without IPTG induction; Lane 2, total protein from E. coli BL21 (DE3) transformed with plasmid pET-32a (+) after induction by IPTG at $37{ }^{\circ} \mathrm{C}$ for $5 \mathrm{~h}$; Lane 3 , total protein from E. coli BL21 (DE3) transformed with plasmid pET-32a $(+)$ without IPTG induction;

Lane 4, total protein from E. coli BL21 (DE3) transformed with plasmid pET-32a (+) after induction by IPTG at $37^{\circ} \mathrm{C}$ for $5 \mathrm{~h}$. B: Lane 5, the purified NLRP3 fusion protein; Lane M, the protein marker. C: Lane 6, Western blot analysis of His-tagged NLRP3 using purified IgG prepared by us. D: Lane 7, Western blot analysis of His-tagged NLRP3 using antibody against with His-tag. Arrowheads indicate the position of the His-tagged NLRP3 protein 


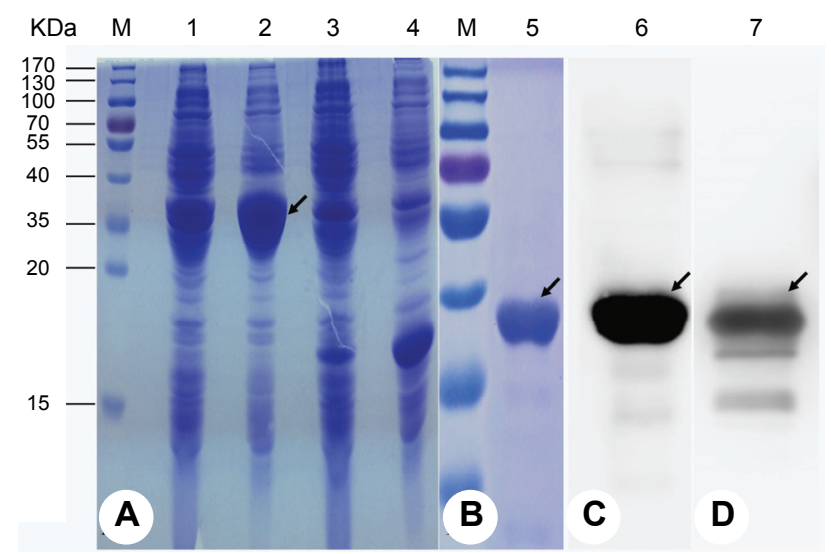

Fig. 4. Analysis of the His-tagged ASC protein expressed in E. coli BL21 (DE3). A: Lane M, the protein marker; Lane 1, total protein from E. coli BL21 (DE3) transformed with plasmid pET-32a (+)-ASC without IPTG induction; Lane 2, total protein from E. coli BL21 (DE3) transformed with plasmid pET-32a (+)-ASC after induction by IPTG at $37^{\circ} \mathrm{C}$ for $5 \mathrm{~h}$; Lane 3, total protein from E. coli BL21 (DE3) transformed with plasmid pET-32a (+) without IPTG induction; Lane 4, total protein from $E$. coli BL21 (DE3) transformed with plasmid pET-32a (+) after induction by IPTG at $37^{\circ} \mathrm{C}$ for $5 \mathrm{~h}$. B: Lane 5, the purified ASC fusion protein; Lane M, the protein marker. C: Lane 6, Western blot analysis of His-tagged ASC using purified IgG prepared by us. D: Lane 7, Western blot analysis of His-tagged ASC using antibody against with His-tag. Arrowheads indicate the position of the His-tagged ASC protein

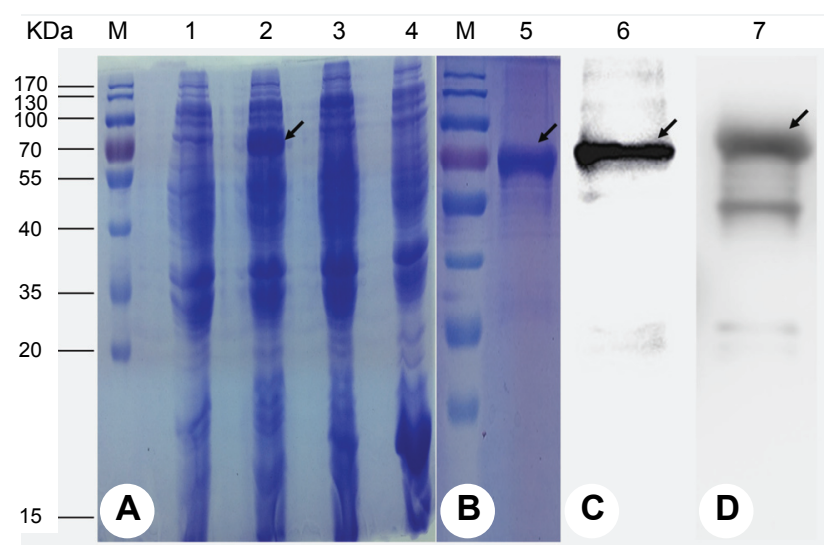

Fig. 5. Analysis of the His-tagged caspase-1 protein expressed in E. coli BL21 (DE3). A: Lane M, the protein marker; Lane 1, total protein from E. coli BL21 (DE3) transformed with plasmid pET-32a (+)-caspase-1 without IPTG induction; Lane 2, total protein from E. coli BL21 (DE3) transformed with plasmid pET-32a (+)-caspase- 1 after induction by IPTG at $37^{\circ} \mathrm{C}$ for $5 \mathrm{~h}$; Lane 3 , total protein from $E$. coli BL21 (DE3) transformed with plasmid pET-32a $(+)$ without IPTG induction; Lane 4, total protein from $E$. coli BL21 (DE3) transformed with plasmid pET-32a (+) after induction by IPTG at $37^{\circ} \mathrm{C}$ for $5 \mathrm{~h}$. B: Lane 5, the purified caspase-1 fusion protein; Lane M, the protein marker. C: Lane 6, Western blot analysis of His-tagged caspase-1 using purified IgG prepared by us. D: Lane 7, Western blot analysis of His-tagged caspase-1 using antibody against with His-tag. Arrowheads indicate the position of the His-tagged caspase-1 protein 


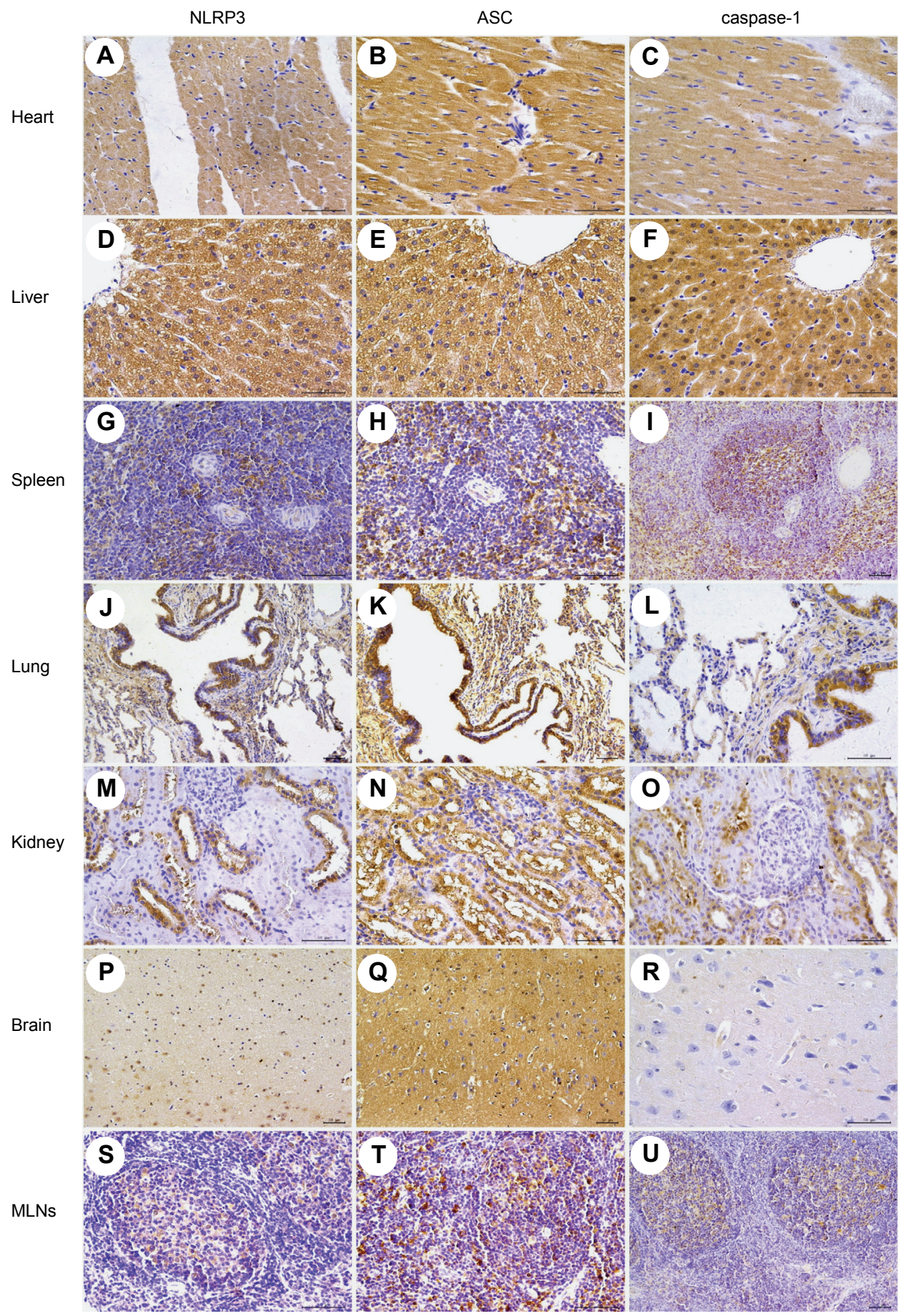




\section{Discussion}

The NLRP3 inflammasome plays an important role in the inflammatory response in infectious diseases. To the best of our knowledge, there is no report about the basic data of gene sequence, expression profile and histological distribution of NLRP3, ASC and caspase-1 in goat. Sequence analysis of these goat genes with other organisms including humans, mice and chickens showed a huge discrepancy which implies either a similarity or a different role. Data of the sequence analysis of NLRP3, ASC and caspase-1 in goat will enrich the data serving for comparison between species. Antigenic determinant analysis suggested that these goat genes differed from those of other organisms except Bos taurus, but a corresponding antibody is lacking and the existing antibodies cannot be used. In this study, we purified the recombinant prokaryotic expression proteins and prepared polyclonal antibodies for NLRP3, ASC and caspase-1 in goat. ELISA and Western blot analysis showed that the prepared antibodies can be used for further research into the function of these genes.

In goat, NLRP3, ASC and caspase-1 were expressed in all tissues examined, as determined by real-time quantitative PCR and immunohistochemical detection. The expression pattern of NLRP3 has been previously reported in several animal species such as human, mouse, pig and chicken (Anderson et al., 2004; Kummer et al., 2007; Tohno et al., 2011; Huang et al., 2014; Ye et al., 2015). The expression of NLRP3 was significantly higher in the spleen than in the heart, liver, lung, kidney, brain and mesenteric lymph nodes of goat, which is consistent with the findings previously reported in the pig (Tohno et al., 2011). However, NLRP3 is most abundant in the trachea and lung of chicken (Ye et al., 2015). Goat NLRP3 is mainly located in cells of the bronchial and bronchiolar epithelium, the renal tubular epithelial cells and the endothelial cells of the germinal centre. Moreover, the hepatocyte nuclei are also positive, but NLRP3 predominantly shows a cytoplasmic staining. Presumably NLRP3 plays a role in both the inflammasome and the nuclear functions. The gene expression profile of ASC showed that ASC mRNA was abundantly expressed in the liver, spleen and kidney; this is consistent with previous reports of ASC being highly expressed in the spleen in humans and mice (Masumoto et al., 1999; Masumoto et al., 2001a, 2001b). Intriguingly, immunohistological analysis of ASC protein expression showed strong positive staining in hepatocytes, focal lymphocytes and renal tubular epithelial cells, which is consistent with the significantly higher expression level

\section{$\leftarrow$}

Fig. 6. Histological distribution of NLRP3, ASC, and caspase-1 in tissues of Hainan black goat. The scale bar is $50 \mu \mathrm{m}$. A, B, and C: heart - NLRP3, ASC and caspase-1, respectively. D, E, and

F: liver - NLRP3, ASC and caspase-1, respectively. G, H, and I: spleen - NLRP3, ASC and caspase-1, respectively. J, K, and L: lung - NLRP3, ASC, and caspase-1, respectively. M, N, and

O: kidney - NLRP3, ASC and caspase-1, respectively. P, Q, and R: brain - NLRP3, ASC and caspase-1, respectively. S, T, and U: MNLs - NLRP3, ASC and caspase-1, respectively 
of mRNA. These data suggest that ASC may participate in the metabolism of substances and host defence against invading pathogens. The mRNA of caspase1 in goat is abundantly expressed in the liver, spleen and MNLs, with a low level in the heart and brain; regarding the spleen, heart and brain this is consistent with data previously reported in humans (Lin et al., 2000). The immunohistochemical results showed that caspase-1 protein was found in the splenic corpuscle and the diffuse lymphoid tissue, which implies a post-translational regulation and a potential mechanism of the host defence response. In addition, the epithelial cells of the bronchi and bronchioles and the renal tubules showed strong positive staining, while the glomeruli and the Bowman's capsules were negative. These organs are the main sites of pathogenesis in many diseases, such as acute respiratory distress syndrome (Dolinay et al., 2012) and infection with bacteria and viruses (Bruno et al., 2012; Bauer et al., 2012). Although there is a significant difference in the expression profiles of NLRP3, ASC and caspase-1 among different species, the largest lymph organ - the spleen - contained abundant amounts of these genes, indicating that they may play a key role in the innate immune and inflammatory response.

The results presented here, showing the gene characteristics, expression profile and tissue distribution of goat NLRP3, ASC and caspase-1, will provide basic data for further investigations into the biological characteristics and functions of the NLRP3 inflammasome, as well as for the prevention and control of inflammatory diseases of goats.

\section{Acknowledgements}

This work was supported by the Science and Technology Planning Project of Guangdong Province (2016A020210079) and the Natural Science Foundation of Guangdong Province (S2013010013041).

\section{References}

Allen, I. C., Scull, M. A., Moore, C. B., Holl, E. K., McElvania-TeKippe, E., Taxman, D. J., Guthrie, E. H., Pickles, R. J. and Ting, J. P. (2009): The NLRP3 inflammasome mediates in vivo innate immunity to influenza A virus through recognition of viral RNA. Immunity $\mathbf{3 0}$, $556-565$.

Anderson, J. P., Mueller, J. L., Rosengren, S., Boyle, D. L., Schaner, P., Cannon, S. B., Goodyear, C. S. and Hoffman, H. M. (2004): Structural, expression, and evolutionary analysis of mouse CIAS1. Gene 338, 25-34.

Bauer, R. N., Brighton, L. E., Mueller, L., Xiang, Z., Rager, J. E., Fry, R. C., Peden, D. B. and Jaspers, I. (2012): Influenza enhances caspase-1 in bronchial epithelial cells from asthmatic volunteers and is associated with pathogenesis. J. Allergy Clin. Immunol. 130, 958-967.

Bruno, S., Grange, C., Collino, F., Deregibus, M. C., Cantaluppi, V., Biancone, L., Tetta, C. and Camussi, G. (2012): Microvesicles derived from mesenchymal stem cells enhance survival in a lethal model of acute kidney injury. PLoS One 7, e33115. 
Chang, C. (2013): The pathogenesis of neonatal autoimmune and autoinflammatory diseases: a comprehensive review. J. Autoimmun. 41, 100-110.

Davis, B. K., Wen, H. and Ting, J. P. (2011): The inflammasome NLRs in immunity, inflammation, and associated diseases. Annu. Rev. Immunol. 29, 707-735.

Dolinay, T., Kim, Y. S., Howrylak, J., Hunninghake, G. M., An, C. H., Fredenburgh, L., Massaro, A. F., Rogers, A., Gazourian, L., Nakahira, K., Haspel, J. A., Landazury, R., Eppanapally, S., Christie, J. D., Meyer, N. J., Ware, L. B., Christiani, D. C., Ryter, S. W., Baron, R. M. and Choi, A. M. (2012): Inflammasome-regulated cytokines are critical mediators of acute lung injury. Am. J. Respir. Crit. Care Med. 185, 1225-1234.

Duan, C., Liao, M., Wang, H., Luo, X., Shao, J., Xu, Y., Li, W., Hao, W. and Luo, S. (2014): Identification, phylogenetic evolutionary analysis of GDQY orf virus isolated from Qingyuan City, Guangdong Province, southern China. Gene 555, 260-268.

Federici, S., Caorsi, R. and Gattorno, M. (2012): The autoinflammatory diseases. Swiss Med. Wkly 142, w13602.

Ferrero-Miliani, L., Nielsen, O. H., Andersen, P. S. and Girardin, S. E. (2007): Chronic inflammation: importance of NOD2 and NALP3 in interleukin-1beta generation. Clin. Exp. Immunol. 147, 227-235.

Horvath, G. L., Schrum, J. E., De Nardo, C. M. and Latz, E. (2011): Intracellular sensing of microbes and danger signals by the inflammasomes. Immunol. Rev. 243, 119-135.

Huang, Z., Yu, M., Tong, S., Jia, K., Liu, R., Wang, H., Li, S. and Ning, Z. (2014): Tissue-specific expression of the NOD-like receptor protein 3 in BALB/c mice. J. Vet. Sci. 15, 173-177.

Joly, S., Ma, N., Sadler, J. J., Soll, D. R., Cassel, S. L. and Sutterwala, F. S. (2009): Cutting edge: Candida albicans hyphae formation triggers activation of the NLRP3 inflammasome. J. Immunol. 183, 3578-3581.

Kanneganti, T. D., Özören, N., Body-Malapel, M., Amer, A., Park, J. H., Franchi, L., Whitfield, J., Barchet, W., Colonna, M., Vandenabeele, P., Bertin, J., Coyle, A., Grant, E. P., Akira, S. and Núñez, G. (2006): Bacterial RNA and small antiviral compounds activate caspase-1 through cryopyrin/NALP3. Nature 440, 233-236.

Konishi, M., Tsuduku, S., Haritani, M., Murakami, K., Tsuboi, T., Kobayashi, C., Yoshikawa, K., Kimura, K. M. and Sentsui, H. (2004): An epidemic of caprine arthritis encephalitis in Japan: isolation of the virus. J. Vet. Med. Sci. 66, 911-917.

Kumar, H., Kawai, T. and Akira, S. (2009): Pathogen recognition in the innate immune response. Biochem. J. 420, 1-16.

Kummer, J. A., Broekhuizen, R., Everett, H., Agostini, L., Kuijk, L., Martinon, F., van Bruggen, R. and Tschopp, J. (2007): Inflammasome components NALP 1 and 3 show distinct but separate expression profiles in human tissues suggesting a site-specific role in the inflammatory response. J. Histochem. Cytochem. 55, 443-452.

Lamkanfi, M. (2011): Emerging inflammasome effector mechanisms. Nat. Rev. Immunol. 11, $213-220$.

Latz, E., Xiao, T. S. and Stutz, A. (2013): Activation and regulation of the inflammasomes. Nat. Rev. Immunol. 13, 397-411.

Li, W., Ning, Z., Hao, W., Song, D., Gao, F., Zhao, K., Liao, X., Li, M., Rock, D. L. and Luo, S. (2012): Isolation and phylogenetic analysis of orf virus from the sheep herd outbreak in northeast China. BMC Vet. Res. 8, 229.

Lin, X. Y., Choi, M. S. and Porter, A. G. (2000): Expression analysis of the human caspase-1 subfamily reveals specific regulation of the CASP5 gene by lipopolysaccharide and interferongamma. J. Biol. Chem. 275, 39920-39926.

Livak, K. J. and Schmittgen, T. D. (2001): Analysis of relative gene expression data using realtime quantitative PCR and the 2(-Delta Delta C(T)) method. Methods 25, 402-408.

Mahla, R. S., Reddy, M. C., Prasad, D. V. and Kumar, H. (2013): Sweeten PAMPs: Role of sugar complexed PAMPs in innate immunity and vaccine biology. Front. Immunol. 4, 248. 
Mariathasan, S., Weiss, D. S., Newton, K., McBride, J., O’Rourke, K., Roose-Girma, M., Lee, W. P., Weinrauch, Y., Monack, D. M. and Dixit, V. M. (2006): Cryopyrin activates the inflammasome in response to toxins and ATP. Nature 440, 228-232.

Martinon, F., Pétrilli, V., Mayor, A., Tardivel, A. and Tschopp, J. (2006): Gout-associated uric acid crystals activate the NALP3 inflammasome. Nature 440, 237-241.

Masumoto, J., Taniguchi, S., Ayukawa, K., Sarvotham, H., Kishino, T., Niikawa, N., Hidaka, E., Katsuyama, T., Higuchi, T. and Sagara, J. (1999): ASC, a novel 22-kDa protein, aggregates during apoptosis of human promyelocytic leukemia HL-60 cells. J. Biol. Chem. 274, 33835-33838.

Masumoto, J., Taniguchi, S., Nakayama, K., Ayukawa, K. and Sagara, J. (2001a): Murine ortholog of ASC, a CARD-containing protein, self-associates and exhibits restricted distribution in developing mouse embryos. Exp. Cell Res. 262, 128-133.

Masumoto, J., Taniguchi, S., Nakayama, J., Shiohara, M., Hidaka, E., Katsuyama, T., Murase, S. and Sagara, J. (2001b): Expression of apoptosis-associated speck-like protein containing a caspase recruitment domain, a pyrin N-terminal homology domain-containing protein, in normal human tissues. J. Histochem. Cytochem. 49, 1269-1275.

Shi, F., Yang, L., Kouadir, M., Yang, Y., Wang, J., Zhou, X., Yin, X. and Zhao, D. (2012): The NALP3 inflammasome is involved in neurotoxic prion peptide-induced microglial activation. J. Neuroinflammation 9, 73.

Sutterwala, F. S., Haasken, S. and Cassel, S. L. (2014): Mechanism of NLRP3 inflammasome activation. Ann. N. Y. Acad. Sci. 1319, 82-95.

Takeuchi, O. and Akira, S. (2010): Pattern recognition receptors and inflammation. Cell 140, 805-820.

Tohno, M., Shimosato, T., Aso, H. and Kitazawa, H. (2011): Immunobiotic Lactobacillus strains augment NLRP3 expression in newborn and adult porcine gut-associated lymphoid tissues. Vet. Immunol. Immunopathol. 144, 410-416.

Venkatesan, G., Balamurugan, V., Singh, R. K. and Bhanuprakash, V. (2010): Goat pox virus isolated from an outbreak at Akola, Maharashtra (India) phylogenetically related to Chinese strain. Trop. Anim. Health Prod. 42, 1053-1056.

Vera-Lastra, O., Medina, G., Cruz-Dominguez, M. P., Jara, L. J. and Shoenfeld, Y. (2013): Autoimmune/inflammatory syndrome induced by adjuvants (Shoenfeld's syndrome): clinical and immunological spectrum. Expert Rev. Clin. Immunol. 9, 361-373.

Wang, D., Zhou, L., Zhou, H., Hou, G., Li, M., Shi, L., Huang, X. and Guan, S. (2014): Effects of nutrition level of concentrate-based diets on growth performance and carcass characteristics of Hainan black goats. Trop. Anim. Health Prod. 46, 783-788.

Wang, D., Zhou, L., Zhou, H., Hou, G., Shi, L., Li, M., Huang, X. and Guan, S. (2015): Effects of nutritional level of concentrate-based diets on meat quality and expression levels of genes related to meat quality in Hainan black goats. Anim. Sci. J. 86, 166-173.

Wu, Y. P., Guan, W. J., Zhao, Q. J., He, X. H., Pu, Y. B., Huo, J. H., Xie, J. F., Han, J. L., Rao, S. Q. and Ma, Y. H. (2009): A fine map for maternal lineage analysis by mitochondrial hypervariable region in 12 Chinese goat breeds. Anim. Sci. J. 80, 372-380.

Ye, J., Yu, M., Zhang, K., Liu, J., Wang, Q., Tao, P., Jia, K., Liao, M. and Ning, Z. (2015): Tissuespecific expression pattern and histological distribution of NLRP3 in Chinese yellow chicken. Vet. Res. Commun. 39, 171-177.

Zhang, K., Xiao, Y., Yu, M., Liu, J., Wang, Q., Tao, P., Liu, S. and Ning, Z. (2016): Phylogenetic analysis of three orf virus strains isolated from different districts in Shandong Province, East China. J. Vet. Med. Sci. 77, 1639-1645.

Zhou, F., Cui, L., Huang, Z., Tong, S., Yu, M., Liu, R. and Ning, Z. (2013): The expression pattern and histological distribution of sialyltransferases ST3Gal III in yellow chicken. Vet. Res. Commun. 37, 285-291. 